American Journal of Biostatistics 1 (1): 42-45, 2010

ISSN 1948-9889

C 2010 Science Publications

\title{
Application of a Statistical Model to Biological Data Analysis: Exclusive Breastfeeding
}

\author{
${ }^{1}$ Naushad Mamode Khan, ${ }^{2}$ Cheika Jahangeer, \\ ${ }^{3}$ Daya Goburdhun and ${ }^{4}$ Maleika Heenaye-Mamode Khan \\ ${ }^{1}$ Department of Mathematics, Faculty of Science, University of Mauritius, Mauritius \\ ${ }^{2}$ School of Health Sciences, University of Pune, India \\ ${ }^{3}$ Faculty of Agriculture, University of Mauritius, Mauritius \\ ${ }^{4}$ Department of Computer Science and Engineering, University of Mauritius, Mauritius
}

\begin{abstract}
Problem statement: Breastfeeding is of utmost importance in the maternal life of a woman, particularly exclusive breastfeeding. Exclusive breastfeeding during the first 6 months of life supports optimal growth and development during infancy and reduces the risk of obliterating diseases and problems. Many probability distributions were proposed to model such data such as the mixed Poisson distributions. However, the estimation methodologies based on such mixed Poisson distributions may be complicated and may not yield consistent and efficient regression estimates. Approach: In this study, we proposed a negative-binomial regression model to analyze the local practices of exclusive breastfeeding and factors affecting this practice. Results: The estimation of parameters is carried out using a quasi-likelihood estimation technique based on a marginal approach via Newton-Raphson iterative procedure. Conclusion: The negative binomial distribution is applied on a sample of data on infant feeding practices in 2006 and has yielded reliable estimates of the regression and overdispersion parameters.
\end{abstract}

Key words: Exclusive breastfeeding, negative binomial, quasi-likelihood, regression, over-dispersion

\section{INTRODUCTION}

The first foremost ideal food for an infant is breast milk and it should meet most of the nutritional requirements if adequately supplied. In fact, the recommended practice by World Health Organization (2003); American Academy of Pediatrics (1997) and Gartner (2005) is exclusive breastfeeding for the first 6 months of life followed by nutritionally adequate and safe complementary foods with continued breastfeeding up to two years of age or beyond.

Moreover, not so recently, one thousand four hundred years ago, the following declaration was promulgated through the Holy Quran "Mothers should suckle their offspring for two whole years" (Surah Baqarah verse 233, Surah Luqman verse 14, Surah Ahqaaf verse 15).

Breast milk is very advantageous in regards to general health, growth and development, while significantly decreasing the risk for a large number of acute and chronic diseases such as respiratory infection, bacterial meningitis and botulism. Many studies have also shown the possible protective effect of human milk feeding against sudden infant death syndrome, insulin dependant diabetes mellitus, Crohn's disease, ulcerative colitis, lymphoma, allergic diseases and chronic digestive diseases (American Academy of Pediatrics, 1997). Moreover, exclusive breastfeeding also improves the motor and language skills as compared to infants who have not been breastfed (Vestergaard et al., 1999). Modernization and the fast changing evolution have led to a decrease in both the incidence and duration of exclusive breastfeeding (Narayan et al., 2005).

Mauritius being a small island in its full swing of development is also affected by this issue where factors such as maternal age, employment, length of maternity leave, place of antenatal treatment, information obtained on breastfeeding, type of delivery and place of delivery have accounted for a decrease in the incidence of exclusive breastfeeding. Moreover, a decline in exclusive breastfeeding pattern has been noticed since 2002 amongst Mauritian mothers, i.e., the prevalence of exclusive breastfeeding at 4 months was 34.2\% in 2002 (Oogarah, 2002). In this study, we use the negativeBinomial regression model (NB) to analyze the practices of exclusive breastfeeding based on a random subset of data collected from a survey on breastfeeding 
in Mauritius over the year 2005-2006. The organization of the study is as follows: We describe the factors influencing exclusive breastfeeding in Mauritius since 2005 and analyze these data using the negative binomial model. Estimation of parameters is done via the quasi-likelihood estimation technique.

Factors influencing exclusive breastfeeding: Maternal age has been regarded as adversely affecting the breastfeeding rates among mothers (Narayan et al., 2005). Employment, maternity leave and the length of maternity leave are very influential on the incidence of exclusive breastfeeding and thus affect mother's choice of feeding practice. Despite the fact that the working mothers may be aware of the advantages of breastfeeding, many of them are rather hesitant to practice exclusive breastfeeding as compared to unemployed mothers. In Mauritius, according to the 2003 report from the Pay Research Bureau, only 12 weeks of maternity leaves are granted to public officers for 3 confinements only (PRB Report, 2003). However, working outside the home and being a full-time worker is related to shorter duration of breastfeeding. Other studies have also reported that one of the most important reasons for mothers to stop breastfeeding at 6 months or earlier was "returning to work" (Integrated Care for Mother and Child, 2004). Many studies have demonstrated that information on breastfeeding can influence a mother's choice of feeding practice. Other authors have stated that health education could improve the present status on infant feeding practices (Singhania et al., 1990). The lack of proper information on breastfeeding sometimes acts as a barrier to its practice though women are strongly determined to breastfeed. It was reiterated that continual support using a nutrition education 'communication mix' is prone to be more effective to result in positive behavior change towards infant feeding practices (Sethi et al., 2003). Support needs to be given to breastfeeding mothers to encourage breastfeeding beyond the first month and also the education of mothers and grandmothers is very important to establish good infant feeding practices (Barton, 2001). The type of delivery may have a negative effect on breastfeeding initiation. Moreover, caesarian section seems to be a big barrier for the rightly timed initiation of breastfeeding. Caesarian section is becoming an increasingly common practice in the private hospitals among the upper and middle income groups and this seems to be an obstacle to successful breastfeeding (Reddy, 1995). The place of antenatal treatment and the place of delivery can also have an impact on the feeding practices of mothers. There are two types of hospital set-up in Mauritius namely the public hospitals and the private hospitals. Both differ in the ways in which antenatal care, perinatal and postnatal care are being provided. Enthusiasm, support and pediatricians involvement are also very essential in the promotion and practice of breastfeeding towards the achievement of optimal infant and child health, growth and development (American Academy of Pediatrics, 1997). A survey was carried out since 2006 with 25,000 mothers having an infant between the age of 5 and 24 months old. We choose a random subset of this data consisting of 6500 mothers. We noted that the average practices of exclusive breastfeeding during the first 6 months of the baby's life is approximately 250 while the variance is 356. This indicates that the data is over-dispersed. To model such data under a regression set-up, we use the negative binomial model. The estimation of parameters is done using the quasi-likelihood estimation technique.

\section{MATERIALS AND METHODS}

We assume $y_{i t}$ follows Negative Binomial distribution (NB) with probability mass function:

$f\left(y_{i}\right)=\frac{\Gamma\left(c^{-1}+y_{i}\right)}{\Gamma\left(c^{-1}\right) y_{i} !}\left(\frac{1}{1+c \theta_{i}}\right)^{c^{-1}}\left(\frac{c \theta_{i}}{1+c \theta_{i}}\right)^{y_{i}}$

i.e.:

$\mathrm{Y}_{\mathrm{i}} \sim \mathrm{NB}\left(1 / \mathrm{c}, \mathrm{c} \theta_{\mathrm{i}}\right)$

where, $\mathrm{c}$ is the over-dispersion parameter and the expectation and variance are given by:

$\mathrm{E}\left(\mathrm{Y}_{\mathrm{i}}\right)=\mathrm{q}_{\mathrm{i}}=\exp \left(\mathrm{x}_{\mathrm{i}}^{\mathrm{T}} \beta\right), \operatorname{Var}\left(\mathrm{Y}_{\mathrm{i}}\right)=\mathrm{q}_{\mathrm{i}}+\mathrm{cq}_{\mathrm{i}}^{2}$

where, c>0. Wedderburn (1974) developed A QuasiLikelihood Estimation technique (QLE) to estimate parameters under generalized linear model. We extend his approach and develop two marginal QLEs under NB. The first QLE is to estimate the vector of regression parameters $\beta$ based on observations $y_{i}$ while the second QLE is to estimate the dispersion index c.

The QLE to estimate $\beta$ is given by:

$\sum_{i=1}^{\mathrm{I}} D_{i, \beta}^{\mathrm{T}} \mathrm{V}_{\mathrm{i}, \beta}^{-1}\left(\mathrm{y}_{\mathrm{i}}-\theta_{\mathrm{i}}\right)=0$

Where:

$\mathrm{V}_{\mathrm{i}, \beta}=\theta_{\mathrm{i}}\left(1+\mathrm{c} \theta_{\mathrm{i}}\right)^{2}$

$\mathrm{D}_{\mathrm{i}, \beta}=\frac{\partial \theta_{\mathrm{i}}}{\partial \beta^{\mathrm{T}}} \theta_{\mathrm{i}} \mathrm{X}_{\mathrm{i}}^{\mathrm{T}}$ is a $\mathrm{p} \times 1$ matrix 
Am. J. Biostatistics 1 (1): 42-45, 2010

The QLE to estimate $\mathrm{c}$ is given by:

$\sum_{\mathrm{i}=1}^{\mathrm{I}} \mathrm{D}_{\mathrm{i}, \alpha}^{\mathrm{T}} \mathrm{V}_{\mathrm{i}, \alpha}^{-1}\left(\mathrm{y}_{\mathrm{i}}^{2}-\eta_{\mathrm{i}}\right)=0$

Where:

$\eta_{\mathrm{i}}=\theta_{\mathrm{i}}\left(1+\mathrm{c} \theta_{\mathrm{i}}\right)+\theta_{\mathrm{i}}^{2}$

$\mathrm{D}_{\mathrm{i}, \alpha}=\theta_{\mathrm{it}}^{2}$

$\mathrm{V}_{\mathrm{i}, \alpha}=$ The variance of $\mathrm{Y}_{\mathrm{i}}^{2}$ and is calculated using:

$\mathrm{V}_{\mathrm{i}, \alpha}=\mathrm{E}\left(\mathrm{Y}_{\mathrm{i}}^{4}\right)-\mathrm{E}\left(\mathrm{Y}_{\mathrm{i}}^{2}\right)^{2}$

where:

$$
\begin{aligned}
\mathrm{V}_{\mathrm{i}, \alpha}= & \theta_{\mathrm{it}}+(6+7 \mathrm{c}) \theta_{\mathrm{it}}^{2}+\left(4+16 \mathrm{c}+12 \mathrm{c}^{2}\right) \theta_{\mathrm{it}}^{3} \\
& +\left(4 \mathrm{c}+10 \mathrm{c}^{2}+6 \mathrm{c}^{3}\right) \theta_{\mathrm{il}}^{4}
\end{aligned}
$$

The iterative equations are given as follows: At the $\mathrm{r}^{\text {th }}$ iteration:

$$
\begin{aligned}
& \left(\hat{\beta}_{r+1}\right)=\left(\hat{\beta}_{r}\right)+\left[\sum_{i=1}^{I} D_{i, \beta}^{T} V_{i, \beta}^{-1} D_{i, \beta}\right]_{r}^{-1}\left[\sum_{i=1}^{1} D_{i, \beta}^{T} V_{i, \beta}^{-1}\left(y_{i}-\theta_{i}\right)\right]_{r} \\
& \left(\hat{c}_{r+1}\right)=\left(\hat{c}_{r}\right)+\left[\sum_{i=1}^{1} D_{i, \alpha}^{T} V_{i, \alpha}^{-1} D_{i, \alpha}\right]_{r}^{-1}\left[\sum_{i=1}^{1} D_{i, \alpha}^{T} V_{i, \alpha}^{-1}\left(y_{i}^{2}-\eta_{i}\right)\right]_{r}
\end{aligned}
$$

Where:

$\hat{\beta}_{\mathrm{r}}$ and $\hat{\mathrm{c}}_{\mathrm{r}}=$ The values of $\hat{\beta}$ and $\hat{c}_{\mathrm{r}}$ at the $\mathrm{r}^{\text {th }}$ iteration

$[.]_{\mathrm{r}} \quad=$ The value of the expression at the $\mathrm{r}^{\text {th }}$ iteration

The estimators are consistent and under mild regularity conditions, for $\mathrm{I} \rightarrow \infty$, it may be shown that $\mathrm{I}^{\frac{1}{2}}((\hat{\beta})-(\beta))^{\mathrm{T}}$ and $\mathrm{I}^{\frac{1}{2}}((\hat{\mathrm{c}})-(\mathrm{c}))^{\mathrm{T}}$ have asymptotic normal distributions. The algorithm to estimate the parameters works as follows: For an initial estimate of $\beta$ and $c$, we iterate Eq. 8 until convergence, then use the updated $\beta$ to update $\mathrm{c}$ in Eq. 9. We then replace the updated $\beta$ and $c$ in Eq. 8 and iterate until convergence. Having obtained the new $\beta$, we replace in Eq. 9 to obtain a new $\alpha$ and the cycle continues until both values converge.

\section{RESULTS}

The covariates are the intercept term, age of the mothers, length of maternity leave, place of antenatal treatment, information on infant feeding practices, type of delivery and place of delivery. These results are obtained by taking small initial values of the regression parameters. The entry in brackets represent the standard errors of each estimate (Table 1).
Table 1: Estimates of the regression parameters based on QLE

\begin{tabular}{lcc}
\multicolumn{3}{c}{ approach for the breastfeeding data: NB model } \\
\hline Intercept & 1.1785 & $(0.3541)$ \\
Age & -2.3211 & $(0.0588)$ \\
Length of maternity leave & 1.2351 & $(0.0525)$ \\
Place of antenatal treatment & -3.2210 & $(0.1871)$ \\
Information & 12.2212 & $(0.0910)$ \\
Type of delivery & -1.2226 & $(0.1111)$ \\
Place of delivery & -2.3044 & $(0.0999)$ \\
$\hat{c}$ & 2.5611 & $(0.1771)$ \\
\hline
\end{tabular}

\section{DISCUSSION}

The negative value of the age factor indicates that age has an adverse effect on the practice of exclusive breastfeeding. The positive estimate of the length of maternity leave shows that as the number of days of maternity leave increases, it is more probable that the mothers will adopt a better infant feeding practice and the incidence of exclusive breastfeeding will increase. The estimated value of the place of antenatal treatment demonstrates the current situation of the private and public health institutions in Mauritius. In the same way, the estimate of the place of delivery is negative because there is a disparity at the level of the private and public health institutions where only the latter have adopted the Baby Friendly Hospital Initiative (BFHI), thereby encouraging proper breastfeeding initiation and successful exclusive breastfeeding for the 6 months. The regression estimate corresponding to the type of delivery indicates that mothers undergoing caesarian section are less likely to practice exclusive breastfeeding. The information parameter estimate justifies that mothers who have been well informed about proper feeding practices are more likely to practice exclusive breastfeeding for the recommended time.

\section{CONCLUSION}

The NB model is a suitable model to analyze breastfeeding data. It has yielded consistent and efficient estimates of the regression and over-dispersion parameters but may not be a suitable model if the value of the mean is greater than the variance of the responses.

\section{REFERENCES}

American Academy of Pediatrics, (AAP), 1997. Work Breastfeeding and the use of human milk. Pediatrics, 100: 1035-1039. DOI: 10.1542/peds.100.6.1035

Barton, S.B., 2001. Infant feeding practices of how low-income rural mothers. Am. J. Maternal Child Nursery, 26: 93-97. 
Gartner, L., 2005. Breastfeeding and the use of human milk (policy statement). Pediatrics, 115: 496-506.

Integrated Care for Mother and Child, 2004. Baby Friendly and Beyond. A Publication of the BabyFriendly Hospital Initiative, USA., pp: 1-6.

Narayan, S., N. Natarajan and K.S. Bawa, 2005. Maternal and neonatal factors adversely affecting breastfeeding in the perinatal period. MJAFI, 61: 216-219. http://medind.nic.in/maa/t05/i3/maat05i3p216.pdf

Oogarah, K.S., 2002. Dissertation on across-sectional study on the infant feeding practices in Mauritius. Dissertation of University of Mauritius.

PRB Report, 2003. General Background and related issues and conditions of service. Minister's Office, Port-Louis, Mauritius. http://www.gov.mu/portal/site/prb/menuitem.bef $8 \mathrm{~b}$ $221 \mathrm{ac} 13 \mathrm{fa} 1 \mathrm{~d} 78 \mathrm{ec} 7 \mathrm{e} 10 \mathrm{a} 0508 \mathrm{a} 0 \mathrm{c} /$ ?content_id=0f828 17868b68010VgnVCM100000ca6a12acRCRD

Reddy, S., 1995. Breastfeeding practices, problems and prospects. J. Fam. Welfare, 41: 43-51.
Sethi, V., S. Kashyap and V. Seth, 2003. Effect of Nutrition education of mothers on infant feeding practices. Indian J. Pediat., 70: 463-466.

Singhania, R., S. Kabra and A. Bansal, 1990. Infant feeing practices in Educated mothers from upper socio-economics status. Indian Pediat., 27: 591-593.

Vestergaard, M., C. Obel, T.B. Henriksen, H.T. Srensen and E. Skajoa et al., 1999. Duration of breastfeeding and development milestones. During the latter half of infancy. Acta Paediat., 88: 1327-1332.

Wedderburn, R., 1974. Quasi-likelihood functions, generalized linear models and the Gauss Newton method. Biometrics, 61: 439-447.

World Health Organization, 2003. Global Strategy for Infant and Young Child Feeding. UNICEF, Geneva, Switzerland, ISBN: 9241562218. 